\title{
EVOLUTIONARY OPTIMIZATION OF CHAOS CONTROL - A NEW APPROACH
}

\author{
Roman Senkerik \\ Ivan Zelinka \\ Department of Applied Informatics \\ Faculty of Applied Informatics, Tomas Bata University in Zlin \\ Nad Stranemi 4511, Zlin 762 72, Czech Republic \\ E-mail: \{ senkerik, zelinka \}@fai.utb.cz
}

\author{
KEYWORDS \\ Chaos, control, optimization, evolutionary algorithms, \\ parameter estimation
}

\begin{abstract}
This research deals with optimization of the control of chaos by means of evolutionary algorithms. The main aim of this work is to show that evolutionary algorithms are capable of the optimization of chaos control and to show a new approach of solving this problem and constructing new cost functions operating in "blackbox mode" without previous exact mathematical analysis of the system, thus without knowledge of stabilizing target state. As a model of deterministic chaotic system, the two dimensional Henon map was used. The optimizations was realized in several ways, each one for another desired periodic orbit. The evolutionary algorithm Self-Organizing Migrating Algorithm (SOMA) was used in four versions. For each version, repeated simulations were conducted to outline the effectiveness and robustness of used method and cost function.
\end{abstract}

\section{INTRODUCTION}

Currently, evolutionary algorithms (EA) are known as powerful tools for almost any difficult and complex optimization problem. But the quality of obtained through the optimizations mostly depends on proper design of the used cost function, especially when the EAs are used for optimization of chaos control. It is well known that deterministic chaos in general and also any technique to control of chaos are sensitive to parameter setting, initial conditions and in the case of optimization they are also extremely sensitive to the construction of used cost function.

This research utilized the Pyragas's delayed feedback control technique (Just 1999, Pyragas 1992). Unlike the original OGY control method (Ott, et al. 1990) it can be simply considered as a targeting and stabilizing algorithm together in one package (Kwon 1999). Another big advantage of Pyragas method is the amount of accessible control parameters. This is very advantageous for successful use of optimization of parameters setting by means of EA, leading to improvement of system behavior and better and faster stabilization to the desired periodic orbits. Some research in this field has been recently done using the evolutionary algorithms for optimization of local control of chaos (Richter and Reinschke, 2000).

This research is concerned with the investigation of the design of the "blackbox mode" cost functions securing the stabilization to desired UPO (unstable periodic orbit) without previous mathematic analysis of system, thus without knowledge of exact UPOs position. The control law is based on Pyragas method: Extended delay feedback control - ETDAS (Pyragas 1995). This research is a continuation of previous experiments with application of EA to chaos control (Zelinka 2007, Senkerik 2007, Senkerik 2006).

\section{PROBLEM DESIGN}

\section{Problem selection and case studies}

The chosen example of a chaotic system was the two dimensional Henon map in form (1).

$$
\begin{aligned}
& x_{n+1}=a-x_{n}^{2}+b y_{n} \\
& y_{n+1}=x_{n}
\end{aligned}
$$

This work primarily consists of three case studies. All of them are focused on an estimation of three accessible control parameters for EDTAS control method to stabilize desired UPO and a comparison of obtained results for used cost function. Desired UPOs are following: $\mathrm{p}-1$ (a fixed point) in the first case, $\mathrm{p}-2$ in the second case and p-4 in the last case. All simulations were 50 times repeated for each EA version. The control method - ETDAS in the discrete form suitable for two dimensional Henon map has the form (2).

$$
\begin{gathered}
x_{n+1}=a-x_{n}^{2}+b y_{n}+F_{n} \\
F_{n}=K\left[(1-R) S_{n-m}-x_{n}\right] \\
S_{n}=x_{n}+R S_{n-m}
\end{gathered}
$$

where $K$ and $R$ are adjustable constants, $F$ is the perturbation, $S$ is given by a delay equation utilizing previous states of the system and $m$ is the period of $m$ periodic orbit to be stabilized. The perturbation $F_{n}$ in 
equations (2) may have an arbitrarily large value, which can cause diverging of the system outside the interval $\{-1.5,1.5\}$. Therefore, $F_{n}$ should have a value between $-F_{\max }, F_{\max }$ and EA should find an appropriate value of this limitation to avoid the diverging of system.

\section{The cost function}

Several unique designs of cost functions (CF) were developed and tested for stabilization of $\mathrm{p}-1$ orbit (fixed point), p- 2 orbit and p-4 orbit. In previous research the $\mathrm{CF}$ had been calculated in general from the distance between desired state (desired UPO) and actual system output on simulation interval $-\tau$. The minimal value of this cost function giving the best solution is zero. The aim of all the simulations was to find the best solution that returns the cost function value as close as possible to zero. This CF is given by (3).

$$
C F=\sum_{t=0}^{\tau}\left|T S_{t}-A S_{t}\right|
$$

where: TS - target state, AS - actual state

Other cost functions ( $\mathrm{CF} 1 \mathrm{CF} 2$ and $\mathrm{CF} 4)$ had to be used for stabilizing of the chaotic system in "blackbox mode" ie. without exact numerical value of target state. In this case it is not possible to use the simple rule of minimizing the area created by the difference between the required and actual state on the whole simulation interval $-\tau$.

Our noval approach is based on searching for periodic orbits in chaotic attractor and stabilizing the system on these periodic orbits by means of applying the optimal value of controller adjustable constants $\left(K, F_{\max }, R\right)$. It means that this new $\mathrm{CF}$ did not take into consideration any numerical target state, but the selected target behavior of system. Therefore the new CFs are based on searching for optimal values of controller parameters securing the stabilization on any type of selected UPO (p-1 orbit - stable state, $\mathrm{p}-2$ orbit - oscillating between two values etc.). The slight disadvantage of this approach is that for each UPO (i.e. different behavior) a different $\mathrm{CF}$ is needed.

The proposal of CF1 used for in the first case ( $\mathrm{p}-1$ orbit) is based on the following simple rule. In discrete systems, the iteration $y(n)$ and $y(n+1)$ of output value must be the same. The idea was to minimize the area created by the difference between the $n$ and $n+1$ output iteration on the whole simulation interval $-\tau$, thus at the same time this proposal of CF should secure fast targeting into the close neighborhood of p-1 orbit and its stabilization. The $\mathrm{CF}_{1}$ has form (4).

$$
C F_{1}=p+\sum_{t=0}^{\tau}|y(n+1)-y(n)|
$$

The next proposal of CF2 used for in the second case (p-2 orbit) is based on following rule. The iteration $y(n)$ and $y(n+2)$ must have the same value. But this rule is also valid for the previous case of $-p-1$ orbit. Thus another condition had to be added. It says that in the case of p-2 orbit there must be some difference between the $n$ and $n+1$ output iteration. Considering the fact of minimizing the $\mathrm{CF}$ value this condition had to be rewritten into this suitable form (5)

$$
\frac{1}{|y(n+1)-y(n)|+c}
$$

where: $c$ - small constant $1.10^{-16}$ which was added to prevent the evolutionary optimization from crashing because upon finding the suboptimal solution stabilized at $\mathrm{p}-1$ orbit it returns the division by zero.

The $\mathrm{CF}_{2}$ has form (6).

$$
C F_{2}=p+\sum_{t=0}^{\tau}|y(n+2)-y(n)|+\frac{1}{|y(n+1)-y(n)|+c}
$$

where: $\mathrm{p}=$ penalization

The last CF used in the third case (p-4 orbit) is based on the following design. The iteration $y(n)$ and $y(n+4)$ must have the same value. But this is also valid for the two previous cases $-\mathrm{p}-1$ and $\mathrm{p}-2$ orbit.

Also in this case another condition had to be added. It states that in case of p-4 orbit there must be some difference between the $n+1$ and $n+3$ output iteration.

The $\mathrm{CF}_{4}$ has form (7).

$$
C F_{4}=p+\sum_{t=0}^{\tau}|y(n+4)-y(n)|+\frac{1}{|y(n+3)-y(n+2)|+c}
$$

where: $\mathrm{p}=$ penalization

In all proposed CFs there had to be included penalization, which should avoid finding of solutions, where the stabilization on saturation boundary values $-1.5,1.5$ or oscillation between them (i.e. artificial p-2 orbit) occurs. This penalization was calculated as the sum of the number of iterations, where the system output reaches the saturation boundary value.

These three "blackbox" modes CFs (6), (6) and (7) are tested in this work. The difference between them can be clearly seen in Fig. 1 - 3, which shows the dependence of CF values on the adjustable parameters $K$ and $F_{\max }$.

where: $\mathrm{p}=$ penalization 


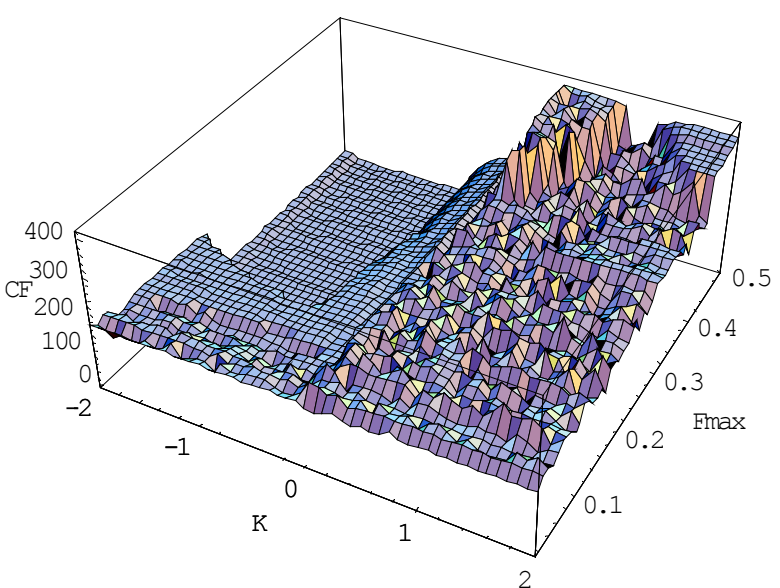

Fig. 1. Dependence of CF value on parameters $K$ and $F_{\max }$ for p-1 orbit, $x_{\text {initial }}=0.7, \mathrm{CF}_{1}, R=0.4426$

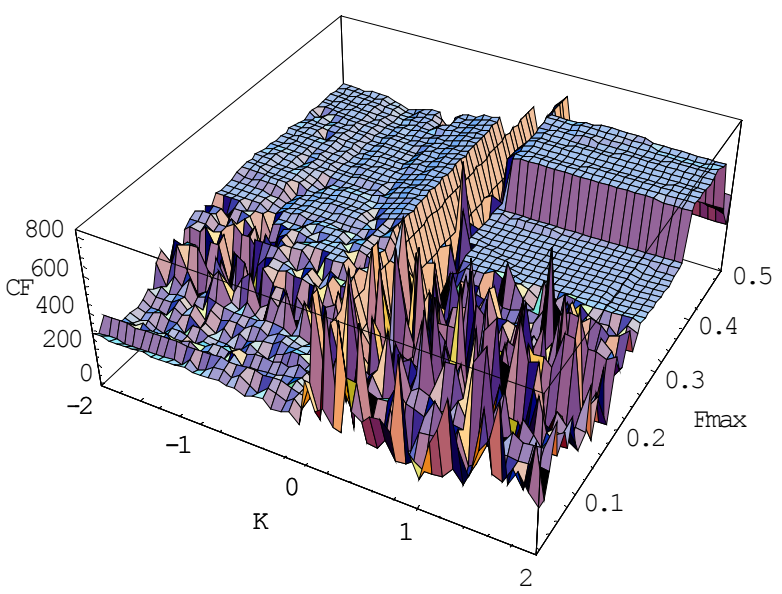

Fig. 2. Dependence of CF value on parameters $K$ and $F_{\max }$ for p-2 orbit, $x_{\text {initial }}=0.7, \mathrm{CF}_{2}, R=0.4387$

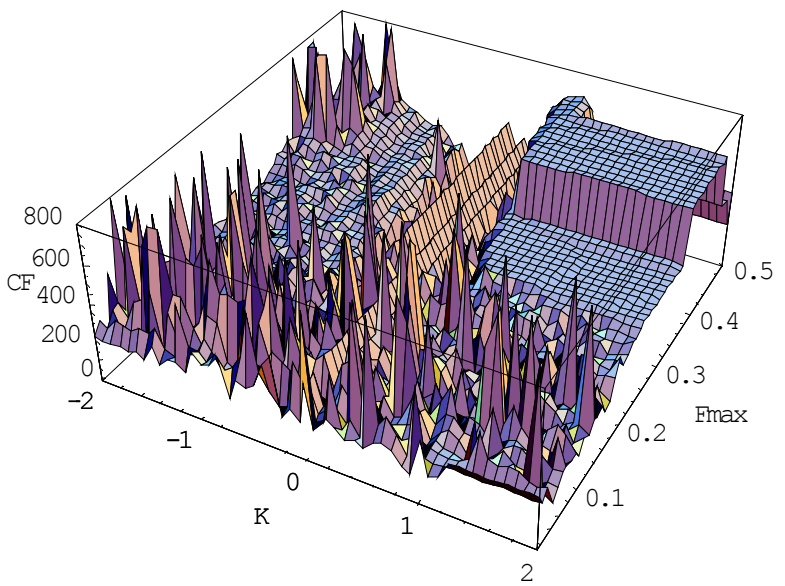

Fig. 3. Dependence of CF value on parameters $K$ and $F_{\max }$ for p-4 orbit, $x_{\text {initial }}=0.7, \mathrm{CF}_{4}, R=0.4574$

\section{Optimization algorithms}

For the experiments described here, stochastic optimization algorithm SOMA (Zelinka, 2004), has been used. It was chosen because it has been proven that this algorithm has the ability to converge towards the global optimum.

\section{EXPERIMENTAL RESULTS}

Four versions of SOMA were used for all simulations. See Table 1 for relation between each version and index corresponding to Tables. Parameters for the optimizing algorithm were set up in such a way in order to reach the same value of maximal CF evaluations for all used versions. Each version of SOMA has been applied 50 times in order to find the actual optimum.

The primary aim here is not to show which version is better or worse but to show that the EA can in reality be used for deterministic chaos control when the "blackbox mode" cost functions are properly defined.

All results are shown only for variable $x$ of two dimensional Henon map because of its form (1), where the variable $y$ has the same values as variable $x$ but it is only phase shifted.

The ranges of all estimated parameters were these:

$$
-2 \leq K \leq 2,0 \leq F_{\max } \leq 0.5 \text { and } 0 \leq R \leq 0.5
$$

Table 1: Used versions of SOMA

\begin{tabular}{|c|c|}
\hline Index & Algorithm / Version \\
\hline 1 & SOMA AllToOne \\
\hline 2 & SOMA AllToRandom \\
\hline 3 & SOMA AllToAll \\
\hline 4 & SOMA AllToAllAdaptive \\
\hline
\end{tabular}

The best solution for each version of SOMA are shown in Tables $2-4$ together with other optimization results like Average IStab value for 50 repeated simulations (Avg. IStab).

\section{Control of chaos, p-1 orbit, $\mathrm{CF}_{1}$}

The first case is focused on the stabilization of $\mathrm{p}-1$ orbit. From previous experiments and numerical analysis of this system, it follows that unperturbed Henon map has this p- 1 orbit: $x_{\mathrm{F}}=0.8$

Each SOMA version gave almost identical result of CF value for the best solution. See Fig. 4 for the best individual solution with the lowest $\mathrm{CF}$ value (SOMA ATAA) and Fig. 5 for the simulation of this best solution with initial conditions uniformly distributed in the region of $0<x_{\text {initial }}<1$ See also Fig. 6 for the simulation of all 200 solutions obtained by all versions of EA. Based on obtained results, it may be stated that the control parameters estimated in the optimizations ensured fast reaching of a desired state without any 
knowledge about its exact position for all 200 simulations. On average, about 90 iterations are required.

Table 2: Results for $\mathrm{p}-1$ orbit, $\mathrm{CF}_{1}$

\begin{tabular}{|c|c|c|c|c|}
\hline EA & 1 & 2 & 3 & 4 \\
\hline$K$ & $-0,6691$ & $-0,6630$ & $-0,7571$ & $-0,6603$ \\
\hline$F_{\max }$ & 0,3172 & 0,3070 & 0,3471 & 0,3195 \\
\hline$R$ & 0,4300 & 0,3961 & 0,4824 & 0,4426 \\
\hline CF Val. & $\mathbf{0 , 3 4 5 9}$ & $\mathbf{0 , 3 4 5 9}$ & $\mathbf{0 , 3 4 8 3}$ & $\mathbf{0 , 3 4 5 9}$ \\
\hline $\begin{array}{c}\text { Avg. } \\
\text { IStab }\end{array}$ & 93 & 99 & 94 & 96 \\
\hline
\end{tabular}

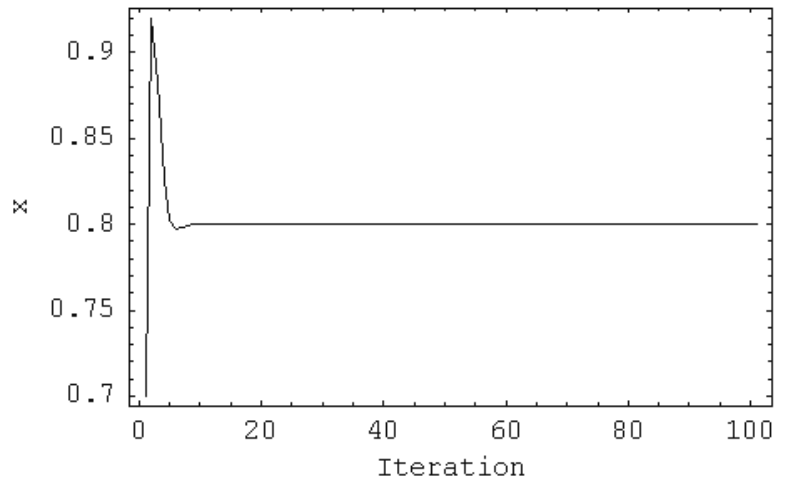

Fig. 4. Best ind. solution: $\mathrm{p}-1$ orbit, $\mathrm{CF}_{1}$, SOMA ATAA

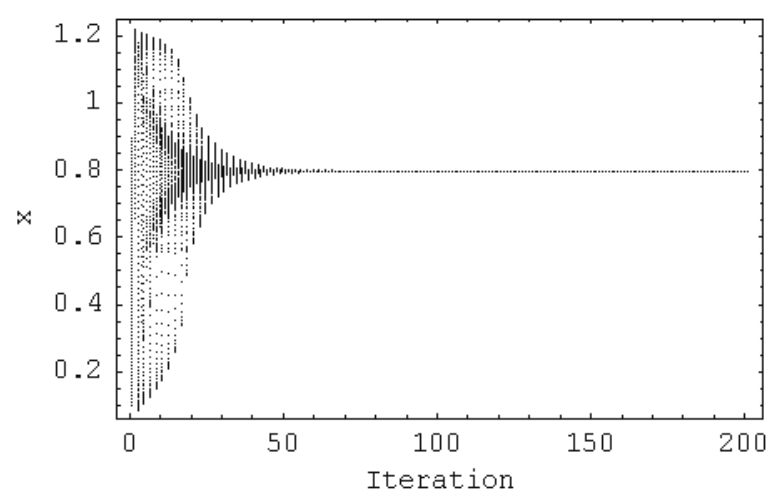

Fig. 5. Best solution: p-1 orbit, $\mathrm{CF}_{1}$, SOMA ATAA, simulation with $0<x_{\text {initial }}<1$.

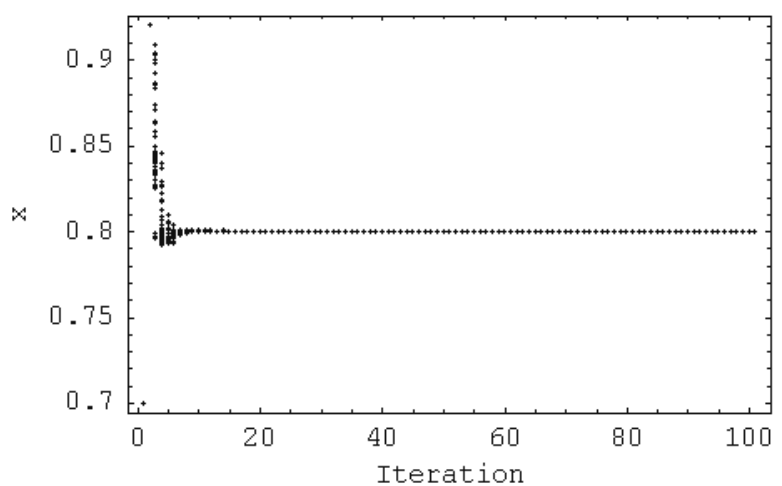

Fig. 6. Simulation of all 200 solutions, $\mathrm{p}-1$ orbit, $\mathrm{CF}_{1}$

\section{Control of chaos, p-2 orbit, $\mathrm{CF}_{2}$}

This case is focused on the stabilization of $2 p$-orbit. Unperturbed Henon map has this p-2 orbit: $x_{1}=-0.56$, $x_{2}=1.26$.

The best results of each SOMA version are given in Table 3. The simulations of the best solution are depicted in Fig. 7 and Fig. 8. As can be seen from Table 3 , three SOMA versions give similar results of the best solution; SOMA ATR has found the lowest CF value. From comparison with previous cases and from Figs. 8 and 9 it follows that the EDTAS control method is not able to reach "exact" stabilization of p-2 orbit. This design of CF secures very fast reaching of desired state but with lower quality of stabilization. More about this problem is written in the special section. For stabilization, on average, about 130 iterations are required.

Table 3: Results for $\mathrm{p}-2$ orbit, $\mathrm{CF}_{2}$

\begin{tabular}{|c|c|c|c|c|}
\hline EA & 1 & 2 & 3 & 4 \\
\hline$K$ & 0,3941 & $-1,4353$ & $-1,4917$ & $-1,4823$ \\
\hline$F_{\max }$ & 0,1345 & 0,2209 & 0,2473 & 0,2622 \\
\hline$R$ & 0,1406 & 0,4387 & 0,4977 & 0,4932 \\
\hline CF Val. & $\mathbf{6 2 , 3 0 6 6}$ & $\mathbf{4 8 , 4 7 6 8}$ & $\mathbf{4 9 , 9 4 0 8}$ & $\mathbf{4 9 , 8 3 5 5}$ \\
\hline $\begin{array}{c}\text { Avg. } \\
\text { IStab }\end{array}$ & 137 & 134 & 137 & 128 \\
\hline
\end{tabular}

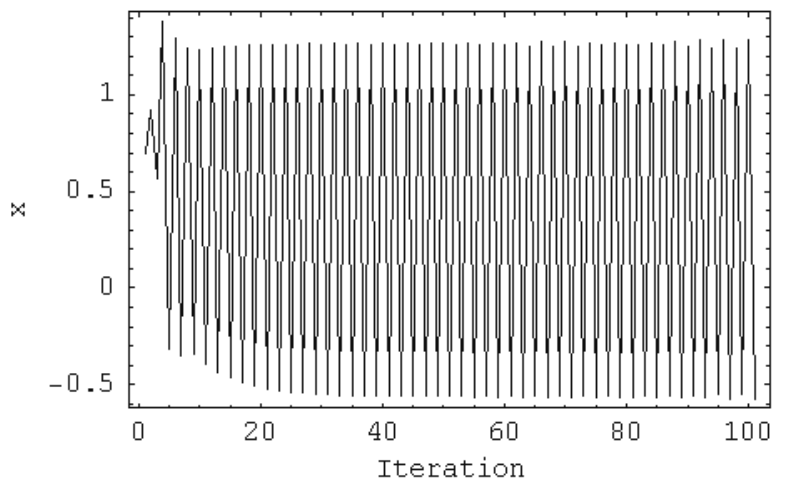

Fig. 7. Best ind. solution: p-2 orbit, $\mathrm{CF}_{2}$, SOMA ATR

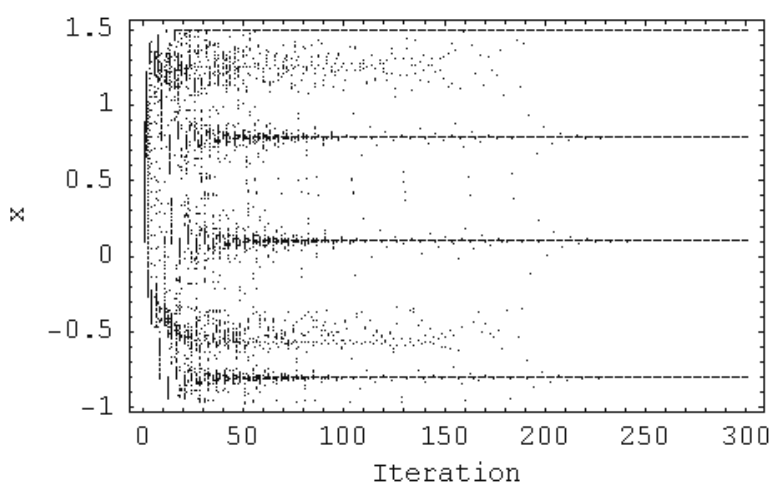

Fig. 8. Best solution: p-2 orbit, $\mathrm{CF}_{2}$, SOMA ATR, simulation with $0<x_{\text {initial }}<1$. 


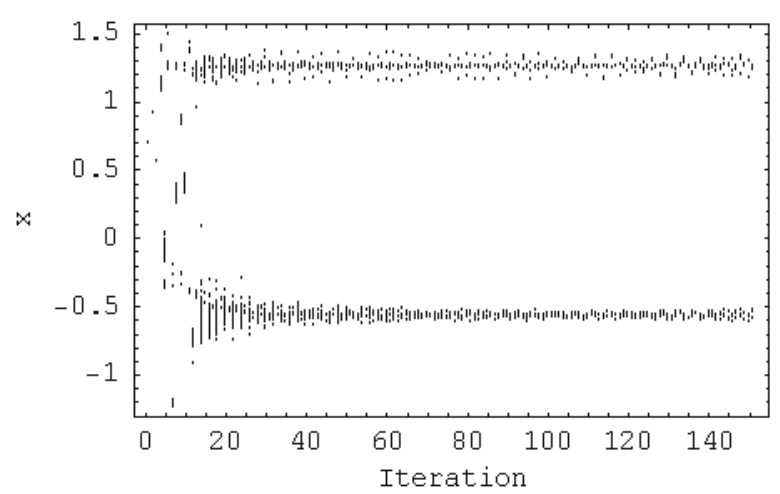

Fig. 9. Simulation of all 200 solutions, $\mathrm{p}-2$ orbit, $\mathrm{CF}_{2}$

\section{Control of chaos, p-4 orbit, $\mathrm{CF}_{4}$}

The last case is focused on the stabilization of $\mathrm{p}-4$ orbit. Unperturbed Henon map has the following p-4 orbit: $x_{1}=0.139, x_{2}=1.4495, x_{3}=-0.8595, x_{4}=0.8962$.

See Table 4 for the results of this optimization. The simulations of the best individual solution are depicted in Figs. 10. and 11. As a conclusion of this case study it is possible to say that all SOMA versions gave almost the same results of the best solution, SOMA ATAA has found the slightly lowest value of CF. From Figs. 10 and 12 follows that the stabilization was reached very quickly and precisely. But this fact is valid only for limited circle of initial conditions (see Fig. 11). For successful stabilization of p-4 orbit, on average, about 145 iterations are needed

Table 4: Results for $\mathrm{p}-4$ orbit, $\mathrm{CF}_{4}$

\begin{tabular}{|c|c|c|c|c|}
\hline EA & 1 & 2 & 3 & 4 \\
\hline$K$ & $-0,3757$ & $-0,4877$ & $-0,3785$ & $-0,3692$ \\
\hline$F_{\max }$ & 0,1152 & 0,1154 & 0,1145 & 0,1163 \\
\hline$R$ & 0,4617 & 0,5074 & 0,4632 & 0,4574 \\
\hline CF Val. & $\mathbf{1 9 , 2 8 5 3}$ & $\mathbf{1 9 , 3 0 0 6}$ & $\mathbf{1 9 , 2 8 9 6}$ & $\mathbf{1 9 , 2 8 3 6}$ \\
\hline $\begin{array}{c}\text { Avg. } \\
\text { IStab }\end{array}$ & 143 & 142 & 143 & 147 \\
\hline
\end{tabular}

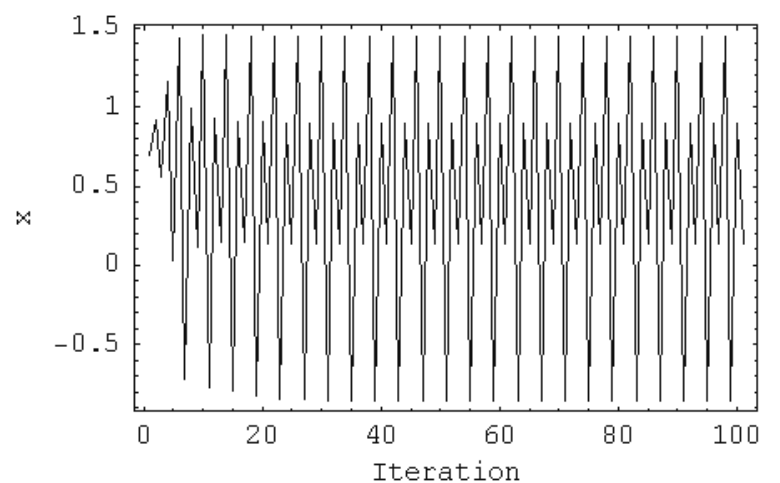

Fig. 10. Best ind. solution: p-4 orbit, $\mathrm{CF}_{4}$, SOMA ATAA

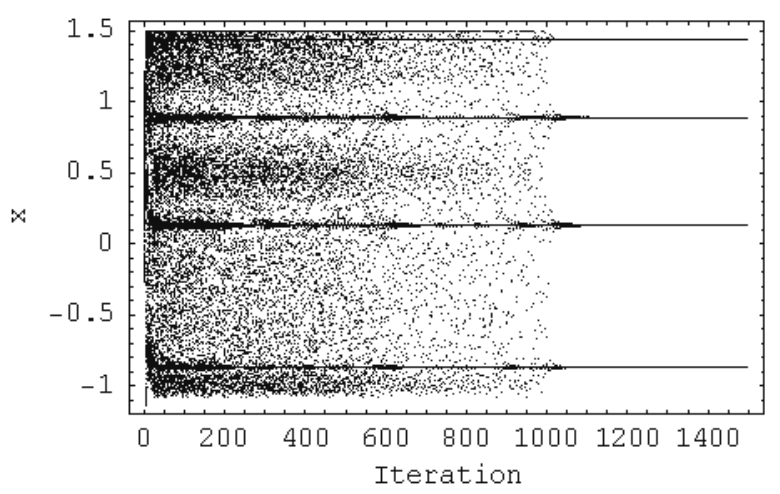

Fig. 11. Best solution: p-4 orbit, $\mathrm{CF}_{4}$, SOMA ATAA, simulation with $0<x_{\text {initial }}<1$.

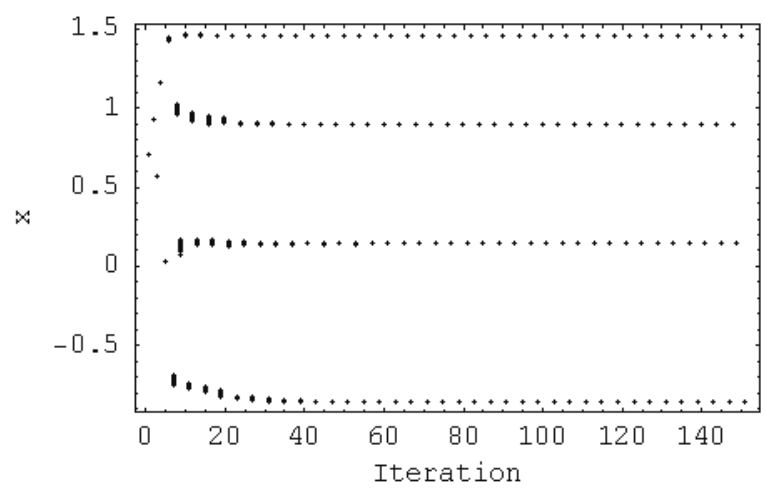

Fig. 12. Simulation of all 200 solutions, p-4 orbit, $\mathrm{CF}_{4}$

\section{Investigation on results of p-2 orbit stabilization}

In the first and last case study, the desired p-orbit was reached rapidly and precisely, but in the second case the EA had found solutions which did not secure such precise stabilization. The answer for this problem can be clearly seen from Figs $13-15$. The first one shows the dependence of $\mathrm{CF}$ value on parameter $K$ ( $F_{\max }=0.2209, R=0.4387$ ). On the left part of this picture there is global optimum CF Value $=48.5$ (see Fig, 14) and on the right positive part there are many local optimums CF value = approx. 62 (see Fig, 15). The global optimum represents solution with very fast but not precise stabilization, whereas the solution given by local optimum secures slow but very precise stabilization on desired $\mathrm{p}-2$ orbit. This problem is caused by including of initial chaotic part into $C F$ value before stabilization. The interesting fact is that version SOMA ATO did not find global optimum while in one or two cases from all 50 runs of other versions the global optimum was found. This can be solved by change of CF design and implementing the experiences with complex $\mathrm{CF}$ designed for higher periodic orbits from previous research. 


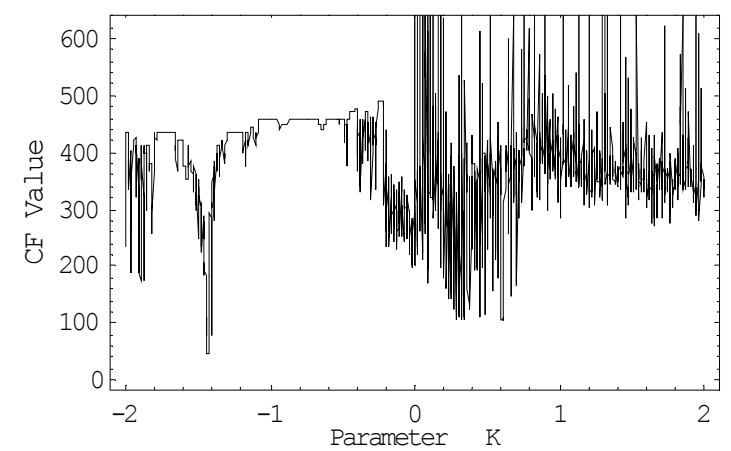

Fig. 13. Dependence of CF Value on $K, \mathrm{p}-2$ orbit

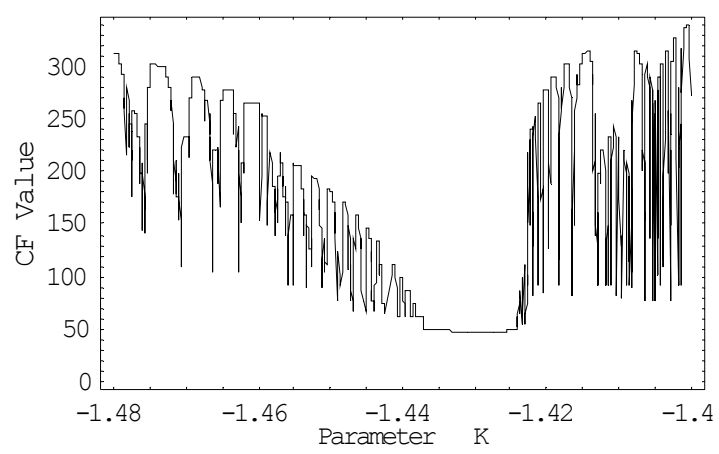

Fig. 14. Dependence of CF Value on $K, \mathrm{p}-2$ orbit

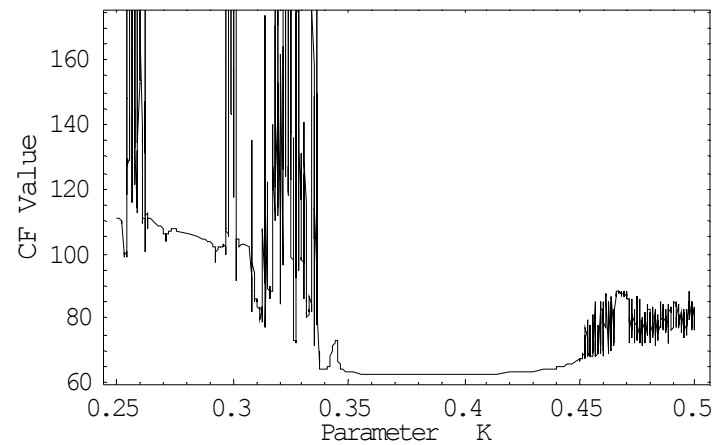

Fig. 15. Dependence of CF Value on $K, \mathrm{p}-2$ orbit

\section{CONCLUSION}

Based on obtained results, it may be claimed that all simulations gave promising results and thus EAs are capable of solving this "blackbox mode" problem. In this research three different CFs were introduced and tested in the task of fast targeting and stabilization of desired periodic orbits. As can be seen from the optimization results presented here, they are extremely sensitive to the construction of the used CF. Any small change in the design of $\mathrm{CF}$ can cause radical improvement of system behavior, but on the other hand can cause worsening of any other observed parameter, as in the case of $\mathrm{CF}_{2}$ for $\mathrm{p}-2$ orbit.

According to all results shown here it is planned that the main activities will be focused on testing more complex cost functions and other criteria for precision distinguishing of stabilized UPO, together with searching for better settings of EA. These promising results gives the possibility for "blackbox mode" evolutionary searching for controller parameters in the task of continuous time systems (Lorenz, Rössler), where the exact mathematical analysis of UPOs position in the chaotic attractor is very demanding.

\section{ACKNOWLEDGEMENT}

This work was supported by the grant NO. MSM 7088352101 of the Ministry of Education of the Czech republic and by grants of Grant Agency of Czech Republic GACR 102/06/1132.

\section{REFERENCES}

Just W., 1999, Principles of Time Delayed Feedback Control, In: Schuster H.G., Handbook of Chaos Control, WileyVch, ISBN 3-527-29436-8.

Kwon O.-J., 1999, Targeting and stabilizing chaotic trajectories in the standard map, Physics Letters A, 258, 229-236.

Ott E., C. Greboki, J.A. Yorke, 1990, Controlling Chaos, Phys. Rev. Lett. 64, 1196-1199.

Pyragas K., 1992, Continuous control of chaos by selfcontrolling feedback, Physics Letters A, 170, 421-428.

Pyragas K., 1995, Control of chaos via extended delay feedback, Physics Letters A, 206, 323-330.

Richter H. and K. J. Reinschke, 2000, Optimization of local control of chaos by an evolutionary algorithm, Physica $D, 144,309-334$.

Zelinka I., 2004, SOMA - Self Organizing Migrating Algorithm, In: New Optimization Techniques in Engineering, (B.V. Babu, G. Onwubolu (eds)), chapter 7, 33, Springer-Verlag, ISBN 3-540-20167X

Zelinka I., Senkerik R., Navratil E, 2007, Investigation on Evolutionary Optimitazion of Chaos Control, CHAOS, SOLITONS \& FRACTALS, doi:10.1016/j.chaos.2007.07.045

Senkerik R., Zelinka I., Navratil E., 2006, Optimization of feedback control of chaos by evolutionary algorithms, in proc $1^{\text {st }}$ IFAC Conference on analysis and control of chaotic systems, Reims, France.

Senkerik R., Zelinka I., Navratil E., 2007, Design of Targeting Cost Function for Evolutionary Optimization of chaos Control, in proc. ECMS 2007, Prague, Czech Republic.

\section{AUTHORS BIOGRAPHIES}

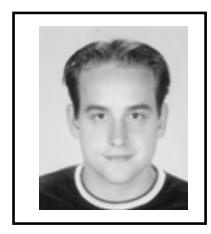

ROMAN SENKERIK was born in the Czech Republic, and went to the Tomas Bata University in Zlin, where he studied Technical Cybernetics and obtained his degree in 2004. He is now a Ph.D. student and lecturer at the same university. Email address: senkerik@fai.utb.cz

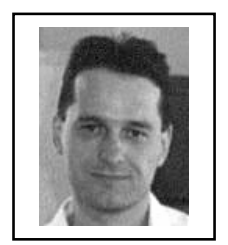

IVAN ZELINKA was born in the Czech Republic, and went to the Technical University of Brno, where he studied Technical Cybernetics and obtained his degree in 1995. He obtained Ph.D. degree in Technical Cybernetics in 2001 at Tomas Bata University in Zlin. Now he is an associated professor (Artificial Intelligence, Theory of Information) and head of department.

Email address: zelinka@fai.utb.cz . 